\title{
Designing Interactive and Motivating Stimuli for Children with Visual Impairments
}

\author{
Florian Güldenpfennig ${ }^{1}$, Peter Fikar², and Roman Ganhör ${ }^{2}$ \\ Vienna University of Technology (TU Wien) \\ ${ }^{1} \mathrm{HCl}$ Group, Argentinierstraße 8, Vienna, Austria \\ ${ }^{2}$ Multidisciplinary Design Group, Favoritenstraße 9-11, Vienna, Austria \\ \{florian.gueldenpfennig, peter.fikar, roman.ganhoer\}@tuwien.ac.at
}

\begin{abstract}
The long-term effects of visual impairments can, in some instances, be mitigated if the sensory cells in the eye are trained appropriately during (early) childhood. Exposure to visual stimuli like cardboards with high-contrast visual patterns is the cornerstone to a successful treatment. However, despite its benefits, this kind of vision training can be tedious and demotivating for children. To leverage training and therapy, we propose interaction design to create multimodal stimuli that are rewarding and motivating. The present work elaborated on this suggestion and presents a design concept that makes the vision therapist a playmaker, coach, playmate, wizard, and statistician all at the same time.
\end{abstract}

Visual impairment. Vision training. Visual stimulus. Interactive toy. Motivation.

\section{INTRODUCTION}

According to the WHO visual impairment is a major health issue concerning the whole population (Cotter et al. 2015; Pascolini \& Mariotti 2011). The reasons for visual disability are manifold and can be located in the peripheral pathway and/or involve a central lesion (Hyvärinen 1995). Often, visual impairment is accompanied by additional handicaps like poor motor skills.

It is crucial for children with these impairments to train their sensory cells as early as possible. That is, the infants or young children should be assessed and exposed to visual stimuli (e.g. specific patterns, strong colours or bright light), allowing their neurons to establish the necessary synapses and increase visual acuity (Hyvärinen 1995). Given the importance of exercising, visual training for affected children is covered by health insurance in many countries. In Austria, the country of this paper's authors, affected children can have two hours of professional vision training each week until the age of seven.

The exercises can, however, be dull and hard to keep up (Linehan et al. 2014). According to the therapists and parents, it is boring for young children to repetitively look at patterns or shapes that they can hardly see.

Hence, therapists and parents attempt to make training sessions as interesting as possible. This includes adapting existing toys or even building custom objects from scratch that feature appropriate sensory properties, for example, highcontrast with respect to the colouring. Figure 1 displays a high-contrast toy doll, which is often used during exercising, because of its horizontal and vertical stripes. The clothing of the doll is specifically designed to meet the (optical) needs of children with low vision.

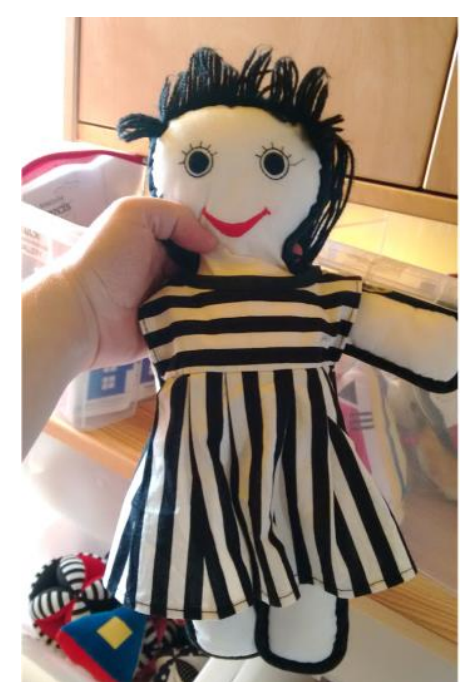

Figure 1: Toy doll as used during vision training wearing a dress with a typical high-contrast, black and white pattern, which enhances perceptibility. While this doll is a popular toy, it nonetheless does not feature any (inter)active elements, which could leverage the child's motivation to engage in vision exercises. 
Many of these customization activities rely on implicit knowledge and best practices. However, as "hacking" skills vary the improved toys and created objects often do not fully meet the intended requirements for visual training (e.g., the object should be motivating to exercise more).

Besides the high prevalence of visual impairments and many opportunities to enhance therapy with smart, interactive stimuli, there is a lack in the $\mathrm{HCl}$ literature examining this particular design space (Linehan et al. 2014). For this reason, we propose to utilize interaction design to create more rewarding and motivating multi-sensory stimuli for affected children. In this course, our focus is on designing interesting, motivating interactions rather than on finding and evaluating novel visual patterns. Basic visual properties and their effect are well documented in the neuropsychological literature. We go on to describe our research approach and present a novel design concept to enhance the conventional visual therapy for young children with visual impairments.

\section{RESEARCH BACKGROUND AND APPROACH}

The work in this paper is part of an applied research project with the primary objective to create interactive therapeutic toys and to leverage conventional therapy. The context of this project includes access to therapists specialised in working with children with visual, and sometimes multiple impairments. In addition to interviewing the therapists, we conduct fieldwork with visually impaired children and their parents.

We follow a user-centred and design-based research agenda. That is, we invest a considerable amount of time and care to study the problem space before we iteratively come up with different design proposals, starting with sketches and eventually leading to fully implemented prototypes.

In the following section, we present Boost Beans, one promising design idea we co-designed with the therapists.

\section{BOOST BEANS: PLAYMAKER, COACH, PLAYMATE, WIZARD, AND STATISTICAN}

The design of Boost Beans draws on the idea to augment everyday objects with interactive technology. It is motivated by observations we made studying the work practices of the therapists. For motivational reasons, therapists incorporated children's favourite toys into the training sessions and these toys acted as rewards (for an example, see Figure 1). However, due to a lack of interactivity, these toys or objects were rarely part of the actual therapy sessions.

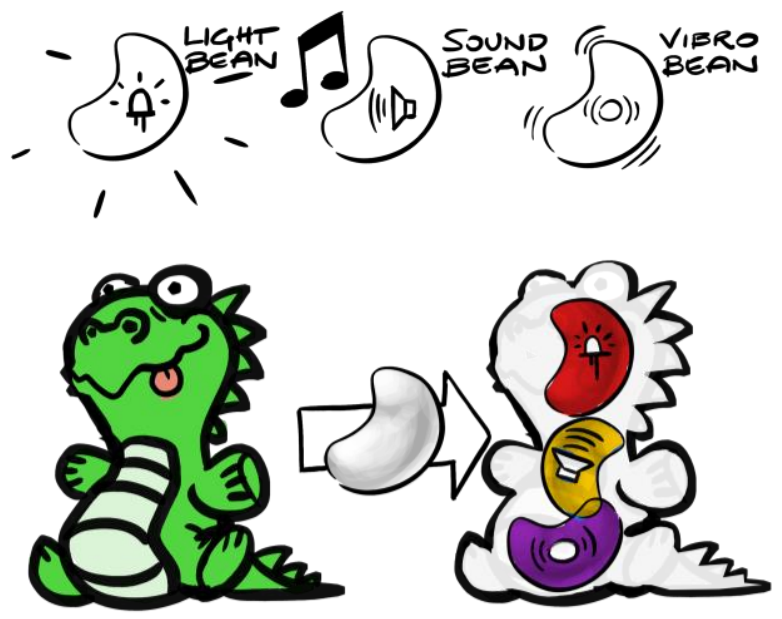

Figure 2: Boost Beans with light, sound and vibration feedback to be put into everyday objects, for example, toys.
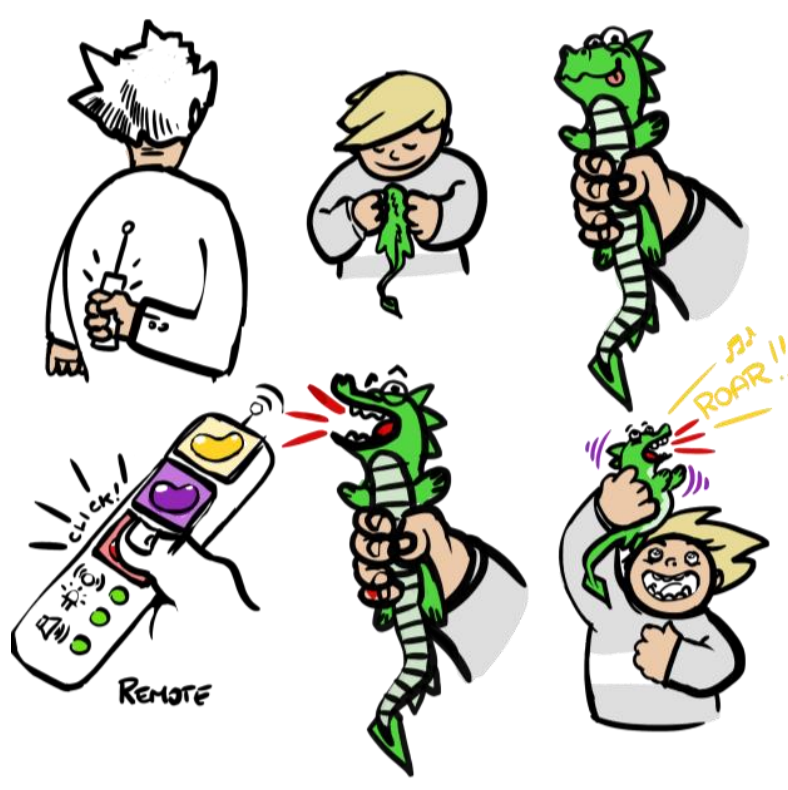

Figure 3: The therapist asks the child to reach out for a specific everyday object. As this object (a dinosaur in the example) is augmented with a remotely controlled Boost Bean, the therapist, acting as the "Wizard" can use the remote control to provide a tactile, acoustic, or visual feedback.

We identified this missed opportunity and developed an interactive means (the Boost Beans) to augment the children's favourite toys or other everyday objects, enabling the therapists to make them a more integral part of the vision training.

The prototype for Boost Beans is comprised of a set of small objects ("beans") that can provide tactile, acoustic, and visual stimuli (see Figure 2). The beans can be triggered with a remote control, and they put the therapist into multiple roles: playmaker, playmate, coach, wizard, and 
statistician. These various roles are outlined in the following.

After selecting a particular therapeutic game and preparing an object or toy by attaching the beans (playmaker), the therapist engages in play with the child (playmate). As they follow their therapeutic agenda, the therapist remains in the position of the trainer or coach. To this end, they motivate the child to identify and reach out for different augmented objects. Should the child touch the corresponding object, the therapist then uses the remote-control to provide appropriate feedback and establishes a two-way interaction. In this way, the therapist takes the role of a "Wizard of Oz" who mimics the objects' interactivity (see Figure 3). Note, a rather "simple" stimulus like a vibration is received as a pleasant and rewarding sensation by many of the children. Whenever operating the remote, the therapist creates a valuable statistic as interactions can optionally be logged. The resulting log file can be utilized for monitoring a child's progress, drawing a more complete picture of the child's development over time.

The scenario provided above should be considered as an example. In the end, it is the therapist who decides how to make best use of the Boost Beans and how to design the therapeutic play exercises.

\section{BOOST BEANS PROTOTYPE}

We have implemented a prototype for Boost Beans using the Arduino platform for controlling the built-in electronic components. Among other things, these included an actuator component, for example, a vibration motor or a speaker, and $433 \mathrm{MHz}$ transceivers for establishing the communication between Boost Bean and remote control. For the

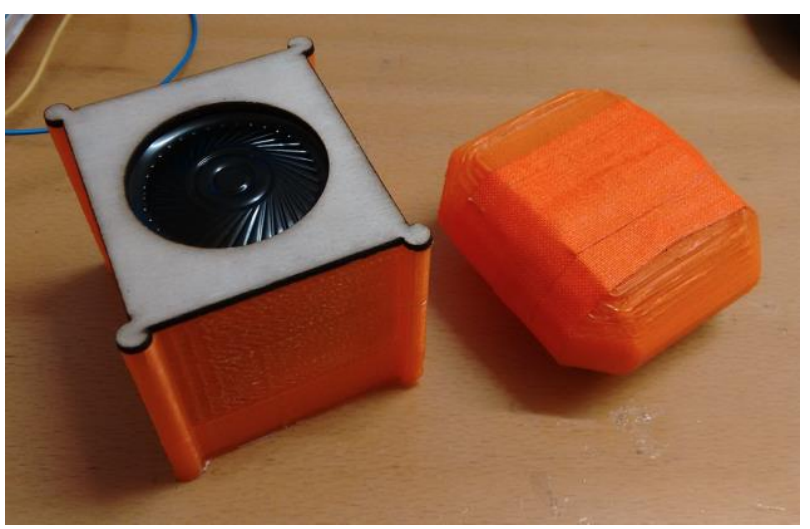

Figure 4: Prototypes for Boost Beans. The device on the left side can be set remotely to record and replay sounds ("Sound Bean"). On the right side, a prototype

for "Vibration Bean" is displayed, which vibrates as long as the corresponding button on the remote control is pressed (not displayed in this image). physical shells of the beans, we used 3D-printing and laser-cutting.

Figure 4 displays two exemplary prototypes for Boost Beans, "Sound Bean" and "Vibration Bean". The first bean can record and replay up to 10 seconds of audio when triggered by the remote control. The second bean contains a vibration motor, amplified by a transistor, to provide tactile feedback. Hence, "Sound Bean" or "Vibration Bean" could be used to make a favourite toy "roar" (see Figure 3) or vibrate when appropriate during the therapeutic play exercise.

As an alternative to the Arduino-powered remote control, we implemented an application for Android based devices for triggering the Boost Beans. This application features a data logger for user interactions, allowing the therapists to later evaluate, which Boost Beans were triggered for how long during exercising.

\section{NEXT STEPS}

We currently reiterate the design of Boost Beans before we will setup an evaluation study of the final version. For example, we will redesign the remote control to make it also usable for visually impaired children as we found that they are eager to play with the "wizard's" remote control. During an early evaluation cycle it turned out that it might be an interesting conceptual 'experiment', to let the children control the beans on their own and not to restrict this role to the therapist.

In the final evaluation, we will have a particular focus on Boost Beans' effect on the children's attention and concentration during exercising. Then again, we are also interested in the amount of distraction the therapists experience when operating the beans remotely. Furthermore, we plan to evaluate the value of the log data for monitoring therapy progress.

\section{CONCLUSION}

In this paper, we presented a concept for augmenting conventional toys and everyday objects with interactivity drawing on small, remotecontrolled devices that we named Boost Beans. The underlying motivation was to animate these everyday objects or toys and thus make them more interesting and motivating as visual stimuli to be used in vision therapy.

With this paper and the illustration of our prototype, we want to draw attention to the under-researched but promising design space of supporting visually impaired children with technology to improve their vision. 


\section{ACKNOWLEDGMENTS}

We would like to thank all of the participants for their valuable time and input.

This research was funded by "Gemeinsame Gesundheitsziele aus dem Rahmen-Pharmavertrag, eine Kooperation von österreichischer Pharmawirtschaft und Sozialversicherung".

\section{REFERENCES}

Cotter, S. et al. (2015). Vision Screening for Children 36 to $<72$ Months: Recommended Practices. Optometry and Vision Science, 92(1), 6-16.

Hyvärinen, L. (1995). Considerations in Evaluation and Treatment of the Child With Low Vision. American $\mathrm{J}$ of Occupational Therapy, 49(9), 891-897.

Linehan, C., et. al (2014). Designing games for the rehabilitation of functional vision for children with cerebral visual impairment. In Proc CHI'14. ACM.

Pascolini, D., \& Mariotti, S. P. (2011). Global estimates of visual impairment: 2010. British Journal of Ophthalmology. 